\title{
Unraveling the hidden complexity of quasideterministic ratchets: Random walks, graphs, and circle maps
}

\author{
Carles Blanch-Mercader $\odot,{ }^{1,2, *}$ Javier G. Orlandi, ${ }^{1,3}$ and Jaume Casademunt ${ }^{1,4, \dagger}$ \\ ${ }^{1}$ Departamento de Física de la Matèria Condensada, University of Barcelona, 08028 Barcelona, Spain \\ ${ }^{2}$ Departament of Biochemistry, University of Geneva, 1211 Geneva, Switzerland \\ ${ }^{3}$ Complexity Science Group, Department of Physics and Astronomy, University of Calgary, Calgary, Canada T2N 1N4 \\ ${ }^{4}$ Universitat de Barcelona Institute of Complex Systems (UBICS), Universitat de Barcelona, Barcelona, Spain
}

(Received 6 September 2018; revised manuscript received 20 March 2019; published 7 January 2020)

\begin{abstract}
Brownian ratchets are shown to feature a nontrivial vanishing-noise limit where the dynamics is reduced to a stochastic alternation between two deterministic circle maps (quasideterministic ratchets). Motivated by cooperative dynamics of molecular motors, here we solve exactly the problem of two interacting quasideterministic ratchets. We show that the dynamics can be described as a random walk on a graph that is specific to each set of parameters. We compute point by point the exact velocity-force $V(f)$ function as a summation over all paths in the specific graph for each $f$, revealing a complex structure that features self-similarity and nontrivial continuity properties. From a general perspective, we unveil that the alternation of two simple piecewise linear circle maps unfolds a very rich variety of dynamical complexity, in particular the phenomenon of piecewise chaos, where chaos emerges from the combination of nonchaotic maps. We show convergence of the finite-noise case to our exact solution.
\end{abstract}

DOI: 10.1103/PhysRevE.101.012203

\section{INTRODUCTION}

Brownian motors play a fundamental role in nonequilibrium statistical physics as a paradigm of directed motion and power generation from noise rectification [1]. The underlying ratchet mechanism, where a particle switches between a state dominated by noise and a symmetry-breaking potential, is a physical realization of the so-called Parrondo's games paradox, a more general phenomenon where the combination of two losing strategies may yield a winning one [2].

The presence of noise is instrumental for the dynamics of single Brownian motors, since both directed motion and power generation are lost for vanishing noise. However, it has been shown that interacting Brownian motors can cooperate to generate a finite power even in the vanishing-noise limit [3]. This paradoxical phenomenon might be at the root of the dramatic cooperative effects reported for assemblies of coupled monomeric kinesin motors [4,5].

In the vanishing-noise limit, power generation for interacting Brownian motors requires two conditions to be satisfied: Individual motors must be able to switch independently between their internal states and the external load must be distributed unevenly on the motors. These two conditions are naturally fulfilled by biological motors pulling on membranebound cargos [4,6-9] but have not yet been implemented in artificial ratchets [10-17]. Independent state switching allows for configurations where a trailing unloaded motor pushes a leading one that is loaded, thus providing a deterministic mechanism for the leading motor to advance against an

\footnotetext{
*Carles.BlanchMercader@unige.ch

†jaume.casademunt@ub.edu
}

external force. In addition, asymmetric loading favors the occurrence of this type of configurations, since unloaded motors are typically faster and thus can easily catch loaded motors. Given the deterministic nature of this mechanism, it makes sense to isolate it by studying the minimal model of two interacting Brownian motors in the vanishing-noise limit.

Solving this problem consists in finding the mean velocity (or other related observables) of the two-motor system for each set of parameters, which in our case reduces to a triad ( $\tau, \sigma, f$ ), with $\tau$ being the ratchet switching time, $\sigma$ being the motor size, and $f$ being the external load. Here we show that the dynamics can be described as a random walk on a directed graph whose topology depends on the parameter triad. The point-by-point exact calculation of the velocityforce function shows that it is nonanalytic in all points and with a complex self-similar geometry at very different scales. In turn, the system dynamics can also be formulated as a stochastic alternation between two deterministic circle maps, specific for each triad. This unveils a rich variety of dynamical regimes, including a novel scenario of piecewise chaotic trajectories. The signature of this hidden chaos manifests in the statistics of two-motor configurations and in their sensitivity to parameters.

\section{THE TWO-MOTOR PROBLEM}

The model we address here is a limiting case of a standard two-state piecewise linear ratchet, discussed in detail, for instance, in Ref. [3]. We consider two particles that interact through a hard-core potential $W(x)$ with a particle size $\sigma$, Fig. 1. An external force $f$ is applied on the leading motor (or particle 1), whereas the trailing motor (or particle 2) is unloaded and thus not subjected to an external force. We 


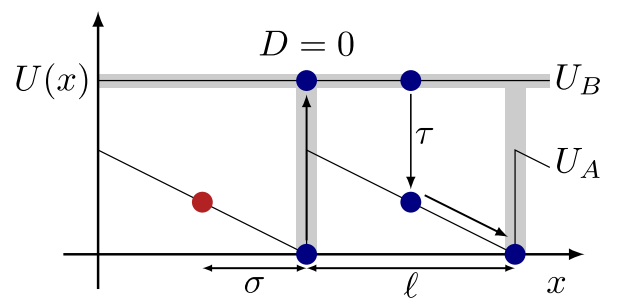

FIG. 1. Schematic of the two-motor problem. A ratchet model with two states: a bound state characterized by an $\ell$-periodic piecewise linear potential $U_{A}$ and a weakly bound state characterized by a flat potential $U_{B}$. The transition $A \rightarrow B$ occurs instantly whenever a particle reaches a $U_{A}$ minima. The transition $B \rightarrow A$ occurs after a time $\tau$. Particles interact through a hard-core potential of size $\sigma$. We take the limit of vanishing thermal fluctuations (i.e., $D=k_{B} T / \lambda \rightarrow$ 0 being $\lambda$ a friction coefficient).

consider a two-state ratchet model that is defined by the set of Langevin equations for the motor positions $x_{i}$,

$$
\lambda \dot{x}_{i}=-U^{\prime}\left(x_{i}, \kappa_{i}\right)-\sum_{j \neq i} W^{\prime}\left(x_{i}-x_{j}\right)-f \delta_{i, 1}+\zeta_{i}(t),
$$

where $\lambda$ is a friction coefficient, $i$ and $j$ denote the particle index, being either 1 or 2 , and $\kappa_{i}$ is a discrete variable that labels the internal state of the $i$ th particle. Each particle can be in two different states: a bound state (or state A) characterized by a piecewise linear $\ell$-periodic potential $U\left(x_{i}, \kappa_{i}=1\right)=$ $U_{A}$ of slope $-U /(\ell-a)$ for $x_{i}<\ell-a$ and slope $U / a$ for $\ell-a<x_{i}<\ell$ or a weakly bound state (or state B) characterized by a flat potential $U\left(x_{i}, \kappa_{i}=0\right)=U_{B}$, Fig. 1 . Switching between states occurs independently for each particle. The transition $A \rightarrow B$ (excitation) occurs instantly, whenever a particle reaches the minimum of $U_{A}$. The transition $B \rightarrow$ $A$ (deexcitation) occurs after a constant lifetime $\tau$. Finally, thermal noise $\zeta_{i}(t)$ is described as a Gaussian white process with autocorrelation

$$
\left\langle\zeta_{i}(t) \zeta_{j}\left(t^{\prime}\right)\right\rangle=2 k_{B} T \lambda \delta_{i j} \delta\left(t-t^{\prime}\right)
$$

where a diffusion coefficient can be naturally defined as $D=$ $k_{B} T / \lambda$.

\section{QUASIDETERMINISTIC RATCHET}

Here we investigate the double limit $a \rightarrow 0$ and $D \rightarrow 0$. When these limits are taken in this order, the indeterminacy associated to particle deexcitations at discontinuities of $U_{A}$ at multiples of $x=\ell$ is resolved. Indeed, excitations $A \rightarrow B$ occurs at $x=\ell$, and subsequently load-free particles diffuse an infinitesimal displacement $\propto \pm \sqrt{D}$ before decaying back to $A$, which will occur with probability $1 / 2$ either on the same spacial period of $U_{A}$ before particle excitation, or on the next period. In the former case, a re-excitation back to state $B$ will occur almost instantly (i.e., after a time $\propto \sqrt{D}$ ). Therefore in the limit, if a transition $B \rightarrow A$ occurs at $x \neq \ell$, a particle will switch to state $A$ with probability 1 , while if a transition $B \rightarrow A$ occurs at $x=\ell$, a particle will either remain at the same position in state $B$ or switch to state $A$ in the next period with probability $1 / 2$ for each outcome. Hence, in the double limit $a \rightarrow 0$ and $D \rightarrow 0$, the stochasticity of the original Brownian dynamics reduces to isolated coin-toss events that punctuate the otherwise deterministic dynamics.

The case of interest here is that of two particles with an opposing external force $f$ applied on the leading one and with hard-core steric interactions of size $\sigma$ (Fig. 1). Henceforth, we restrict the study to $0<f<1$ and exclude net backward motion of the leading particle. Taking $\ell=\lambda=-U_{A}^{\prime}=1$, we have $U_{A}(x)=-(x-[x])$ and $U_{B}=$ const. Due to translation invariance, we set the position origin $x=0$ at a discontinuity of the potential $U_{A}$, and due to its spatial periodicity, we define particle positions modulo 1 . The leading particle 1 moves with a velocity $-\left(U^{\prime}+f\right)$ and the trailing particle 2 with a velocity $-U^{\prime}$, where $U^{\prime}$ is either $U_{A}^{\prime}=-1$ or $U_{B}^{\prime}=0$. When the two particles are in contact, at a distance $\sigma$, they both move with the arithmetic mean of the corresponding velocity.

Under the double limit $a \rightarrow 0$ and $D \rightarrow 0$, Eqs. (1) describing the dynamics of two interacting Brownian ratchets reduce exactly to a simple set of rules for the two position variables $x_{i}(t)$ (with $x_{1} \geqslant x_{2}+\sigma$ ), and two discrete state variables $\kappa_{i}(t)=0,1$ (respectively for $U_{B}$ and $U_{A}$ ). It is convenient to introduce two auxiliary continuous variables $\theta_{i}$, which account for the internal clock that controls the time in the $U_{B}$ state. We define that particles are in contact, if both $x_{1}-x_{2}=\sigma$ and $\kappa_{1}-\kappa_{2}-f \leqslant 0$, meaning that the relative speed would be $\dot{x}_{1}-\dot{x}_{2}<0$ if the two particles were not interacting. The resulting set of rules are, for $i=1,2$ :

(a) $\dot{x}_{i}=\kappa_{i}-\delta_{i 1} f$ (if not in contact)

(b) $\dot{x}_{i}=\left(\kappa_{1}+\kappa_{2}-f\right) / 2$ (if in contact)

(c) $\dot{\theta}_{i}=\left(1-\kappa_{i}\right) / \tau$

(d) if $x_{i}\left(t_{n}\right)=n$ with $x_{i}\left(t_{n}^{-}\right) \neq n$ and $\kappa_{i}\left(t_{n}^{-}\right)=1$,

then $\kappa_{i}\left(t_{n}\right)=0$

(e) if $\theta_{i}\left(t_{m}\right)=m$ with $\theta_{i}\left(t_{m}^{-}\right) \neq m$ and $\kappa_{i}\left(t_{m}^{-}\right)=0$, then

(e1) if $x_{i}\left(t_{m}\right) \neq p$, then $\kappa_{i}\left(t_{m}\right)=1$

(e2) if $x_{i}\left(t_{m}\right)=p$, then $\kappa_{i}\left(t_{m}\right)=0$ or 1 , with prob $1 / 2$,

where $n, m, p$ are any integer numbers, $t^{-} \equiv t-0^{+}$and $t_{n}, t_{m}$ stand for the generic transition times of $\kappa$. Rule (d) expresses that the transition $\kappa=1 \rightarrow 0$ occurs whenever the particle reaches the minimum of $U_{A}$. Rule (e) expresses that the transition $\kappa=0 \rightarrow 1$ occurs whenever a cycle of duration $\tau$ is completed, provided that the particle is not at a discontinuity of $U_{A}$, in which case the transition $\kappa=0 \rightarrow 1$ occurs with probability $1 / 2$. We call quasideterministic ratchet to the simple stochastic set of rules that describe exactly the dynamics of two interacting Brownian motors given by Eqs. (1) in the double limit $a \rightarrow 0$ and $D \rightarrow 0$.

\section{RANDOM WALKS ON DIRECTED GRAPHS}

We assign a graph to each triad $(\tau, \sigma, f)$ by associating a node to the configuration of the system at the instant a stochastic event (e2) takes place. Most frequently, particle 2 undergoes events (e2) since its deexcitations largely occur at discontinuities of state $U_{A}$, unless particle 1 pushes particle 2 backward. An arrow connects two nodes if a deterministic trajectory connects two events (e2). Hence, each node originates two arrows, targeting to the two nodes that are deterministically connected by the two outcomes of (e2). The successive branching of the evolution due to events (e2) complete the graph (see Fig. 2 or supplemental Movies 1-3 [18]). 
(a)

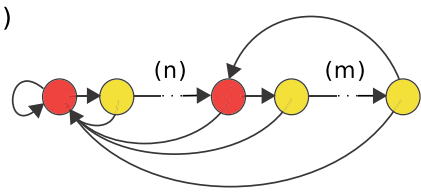

(c)

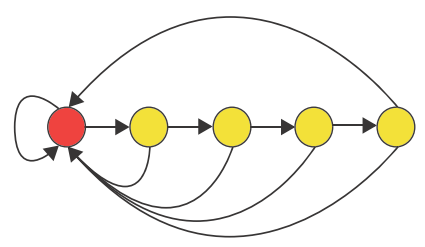

(b)

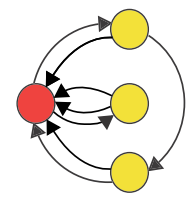

(d)

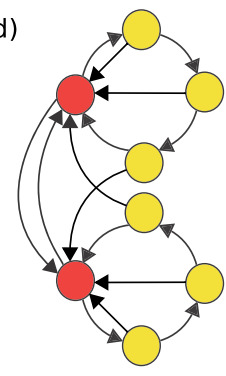

FIG. 2. Directed graphs of the two-motor problem. (a) Generic graph in the regime of sufficiently small $f$ and $\tau$, such as the regime where both $f \tau<1-\sigma$ and $\tau<2 \sigma /(1-f)$ are satisfied, where $n$ and $m$ are integers. (b) Graph corresponding to $(\tau, \sigma, f)=$ $(1,1 / 3,1 / 3)$, where particle 1 undergoes an event (e2). (c) Graph corresponding to $(\tau, \sigma, f)=(1,1 / 3,3 / 5)$ that is within the regime in (a). (d) Graph corresponding to $(\tau, \sigma, f)=(1,1 / 3,1 / 4)$ that is away from the regime in (a). The nodes with only one incoming arrow are yellow and otherwise are red.

The configuration where the two particles slide down together until the minimum of the potential $U_{A}\left(\kappa_{1}=\kappa_{2}=1\right)$, reaching $x_{1}=1^{-}$and $x_{2}=1^{-}-\sigma$, defines the underlying (deterministic) cooperative mechanism of the two-motor problem, since particle 1 is able to hop forward to the next period by being pushed by particle 2 . The system recurrently visits this configuration, since only particle 2 can advance a period by itself. We thus establish the graph origin at the first node encountered after this configuration.

A simple class of graphs that is generic for sufficiently small $f$ and $\tau$, such as the regime where both $f \tau<1-\sigma$ and $\tau<2 \sigma /(1-f)$ are satisfied, is plotted in Fig. 2(a). To understand how is this graph generated, consider first those trajectories for which the outcome of events (e2) for particle 2 are enforced to $\kappa_{2}=0$. In this case, particle 1 trajectory is restricted within a period of the potential $U_{A}$. Indeed, after some possible $n$ steps sliding down the potential $U_{A}$, particle 1 will repeat the following cycle: From $\kappa_{1}=0$ and $x_{1}$ integer, it will move backward a distance $\tau f$ at a velocity $-f$, and then it switches from $B$ to $A$, slides down $U_{A}$ at a velocity $1-f>0$ and a distance $\tau f$, and on reaching the minimum of $U_{A}$, switches back to $\kappa_{1}=0$ and $x_{1}$ integer (see also Appendix A). The total duration of this cycle is $T=\tau /(1-f)$. Therefore, the successive graph nodes will be determined by the positions of particle 1 at those instants when particle 2 undergoes an event (e2) or equivalently at times multiple of $\tau$. When $f$ is rational, the node number of this cycle $m$ is determined by the commensurability condition $p T=\tau m$, where $m$ and $p$ are integers; otherwise, the node number is infinite. On the other hand, let us consider those trajectories for which the outcomes of events (e2) for particle 2 correspond to $\kappa_{2}=1$. In this case, particle 2 will deterministically push particle 1 to the next period of the potential $U_{A}$, and thus the system will return to the graph origin. Therefore, each node of the graph in Fig. 2(a) will be connected to the graph origin, completing the graph.

Graph nodes have typically one incoming arrow (yellow nodes in Fig. 2), but some frequently visited nodes, such as the graph origin, may have many (red nodes in Fig. 2). The total number of nodes could be finite or infinite depending on parameters. In general, graphs are planar (embeddable in a $2 d$ space) and ergodic (all nodes visited in a finite time). The graph topology, meaning their set of connections and nodes, is specific to each parameter triad $(\tau, \sigma, f)$. Therefore, different parameter regimes correspond to different graph topologies; other illustrative examples of graphs are shown in Figs. 2(b)-2(d) or in supplemental Movies 1-3 [18].

Each arrow in the graph can be weighted by the deterministic time lapse and distance traveled (either 0 or 1) between the two connected nodes. In addition, for a given closed path $n_{i}$ in the graph we can compute: the corresponding probability $\mathcal{P}_{\left[n_{i}\right]}$, the total path duration $\mathcal{T}_{\left[n_{i}\right]}$, and the total distance traveled $\mathcal{N}_{\left[n_{i}\right]}$. Then physical observables such as the mean velocity $V$ take the form of discrete path integrals,

$$
V=\frac{\sum_{\left[n_{i}\right]} \mathcal{N}_{\left[n_{i}\right]} \mathcal{P}_{\left[n_{i}\right]}}{\sum_{\left[n_{i}\right]} \mathcal{T}_{\left[n_{i}\right]} \mathcal{P}_{\left[n_{i}\right]}}
$$

Adding the total variation of $\theta_{1}+\theta_{2}, \Theta_{\left[n_{i}\right]}$, which corresponds to the total time both particles spent in state $B$, allows us to introduce new physical observables such as the mean work per motor excitation $w$ that reads

$$
w=f \tau \frac{\sum_{\left[n_{i}\right]} \mathcal{N}_{\left[n_{i}\right]} \mathcal{P}_{\left[n_{i}\right]}}{\sum_{\left[n_{i}\right]} \Theta_{\left[n_{i}\right]} \mathcal{P}_{\left[n_{i}\right]}} .
$$

Figure 3 shows the velocity-force function $V(f)$ for a choice of $\sigma$ and $\tau$, obtained from Eq. (3). $V$ is computed with infinite precision (rational numbers) for $10^{7}$ points in the first panel of Fig. 3, $10^{8}$ in the firsts zooms, and $10^{9}$ in the second zooms. We use the GNU multiple precision arithmetic library to compute the exact path integrals. As long as the parameters are all rational, the graphs had typically fewer than 300 nodes and the number of paths in the graph remained finite. Some graphs had a number of paths so large that it hindered the exact summation of path integrals (for $f<1 / 3$ in Fig. 3), and in those cases, Monte Carlo techniques were used to compute $V$ as a time-averaged velocity of long trajectories. Figure 3 reveals intricate self-similar structures that emerge at many different scales. Graphs with large numbers of nodes were required to resolve the structure of $V$ in regions of complex fine structure. Discontinuities are expected whenever the graph topology changes. Discontinuities are clear to the eye for those graphs with small number of nodes, such as $f=$ 1/3, Fig. 3. Remarkably, for some parameter regions, such as for both $f \tau<1-\sigma$ and $\tau<2 \sigma /(1-f)$ are satisfied, we found that the set of discontinuities has zero measure and that $V(f)$ is continuous within a finite-measure subset of irrational values for $f$ and real values for $\sigma$ and $\tau$, for which the graph topology is locally preserved (see Appendix B).

\section{CIRCLE MAPS AND HIDDEN CHAOS}

The condition (e2) for particle 2 that defines graph nodes (i.e., $\theta_{2}=m, x_{2}=n$ and $\kappa_{2}=0$ for any integers $m, n$ ), defines also a Poincaré section for the evolution of particle 1 , 


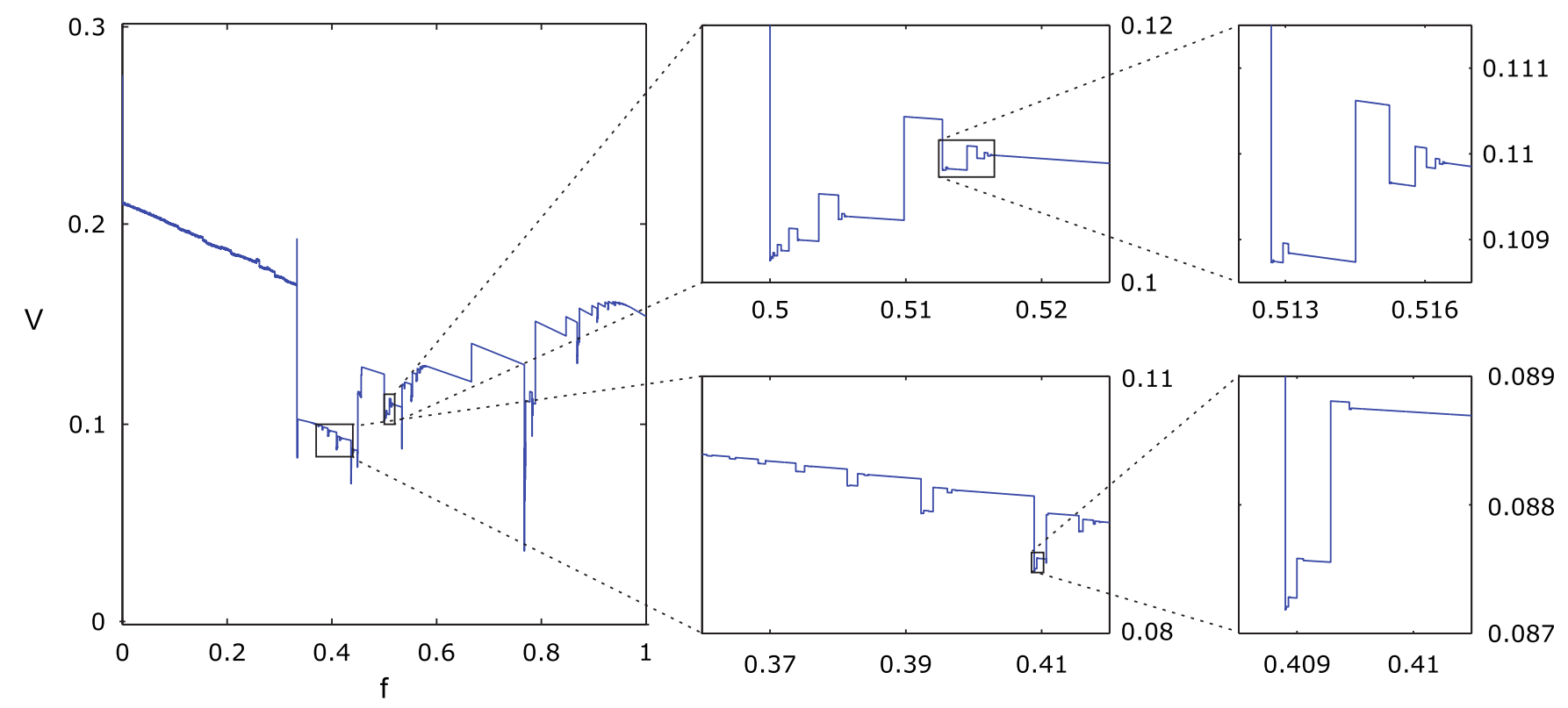

FIG. 3. Leftmost panel: Velocity-force $V(f)$ function for $\sigma=1 / 2$ and $\tau=3 / 2$. Four rightmost panels: Magnifications of the corresponding solid squared black contours. The values of the velocity given by Eq. (3) are computed as exact fractions (infinite precision) for rational values of $f$, except for the range $f \in(0,1 / 3)$ in which the velocity is approximate by the time-averaged velocity of long trajectories from the two-motor problem. The points are connected by lines for better visualization.

allowing us to construct a pair of circle maps for describing the evolution of the system.

A circle map is a transformation from a circle into itself. For $f \neq 0$, particle 1 is unable to advance to the next period by itself. Therefore, the accessible positions for particle 1 [those for which a transition (e2) of particle 2 can occur] belong to a bounded interval $\left(x_{1}, \kappa_{1}\right) \in\left[x_{0}, 1\right] \times\{0,1\}$, where $\sigma \leqslant$ $x_{0} \leqslant 1$ and $x_{0}$ depends on parameters. These configurations of particle 1 can be conveniently parametrized by a single degree of freedom: $t\left(x_{1}, \kappa_{1}\right)$ defined as the time $t$ required for particle 1 to reach $\left(x_{1}, \kappa_{1}\right)$ starting at $\left(x_{0}, 1\right)$ without being in contact with particle 2 (see Appendix A). $T$ corresponds to the maximum of $t\left(x_{1}, \kappa_{1}\right)$, which generally is set by $T=t\left(x_{0}, 0\right)$ with $0 \leqslant t<T$. Therefore, the set of accessible configurations for particle 1 is homeomorphic to a circle.
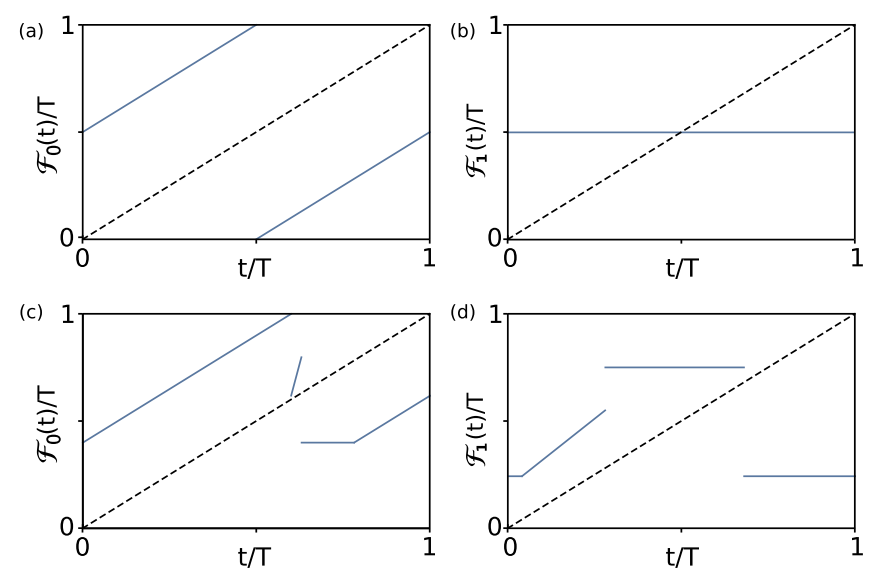

FIG. 4. (a) and (c) Circle map $\mathcal{F}_{0}$ for $(\tau, \sigma, f)=(1,1 / 3,1 / 2)$ and $(1,19 / 40,7 / 10)$, respectively. (b) and (d) Circle map $\mathcal{F}_{1}$ for $(\tau, \sigma, f)=(1,1 / 2,1 / 6)$ and $(2,1 / 2,1 / 5)$, respectively.
The new parametrization $t\left(x_{1}, \kappa_{1}\right)$ allows to interpret the time evolution of the two-motor problem as a stochastic alternation between two circle maps $\mathcal{F}_{0}$ and $\mathcal{F}_{1}$, corresponding to the two possible deterministic evolutions of particle 1 that depend on whether particles 2 switches to $A\left(\kappa_{2}=1\right)$ or remains at $B\left(\kappa_{2}=0\right)$ when an event (e2) occurs. A realization of the stochastic dynamics of the system is, therefore, described by a random string of zeros and ones $\left(\alpha_{1}, \alpha_{2}, \ldots\right)$ with $\alpha_{i}=0$ or 1 , such that $t_{n+1}=\alpha_{n} \mathcal{F}_{1}\left(t_{n}\right)+\left(1-\alpha_{n}\right) \mathcal{F}_{0}\left(t_{n}\right)$, where $t_{n+1}$ and $t_{n}$ stands for two accessible configurations between two consecutive events (e2) for particle 2 .

The circle maps $\mathcal{F}_{0}$ and $\mathcal{F}_{1}$ can be derived analytically for all parameter regimes; some explicit examples are shown in Fig. 4 and Appendix C. Both circle maps are piecewise linear with segments of finite slope and segments with zero slope. The phenomenology of these maps has been studied in detail in Ref. [19-21], displaying complex bifurcation diagrams.

The periodic orbits of $\mathcal{F}_{0,1}$ are deeply connected to the topology of graphs and by extension to the physical observables of the two-motor problem. For instance, for $f>1 / 3$ in Fig. 3, the number of graph nodes equals the period of the corresponding periodic orbits of $\mathcal{F}_{0}$, since $\mathcal{F}_{1}=$ const (see Appendix C). Figure 5 shows the bifurcation diagram of graph nodes as a function of $\tau$, which exhibits a complex organization with broad intervals where the number of nodes remains constant and narrow intervals where the nodes arrange self-similarly. Therefore, complex attributes of node arrangements can be directly correlated to characteristic features of the underlying piecewise linear circle maps $\mathcal{F}_{0,1}$, such as mode-locking, or the so-called period incrementing scenarios or nested period incrementing scenarios [20,21]. Similar motifs are observed in the velocity-force curve Fig. 3. In particular, we find that at the same force intervals, there is a correlation between self-similar structures of $V(f)$ in Fig. 3 for $f>4 / 5$, and the self-similar arrangements of orbits of 


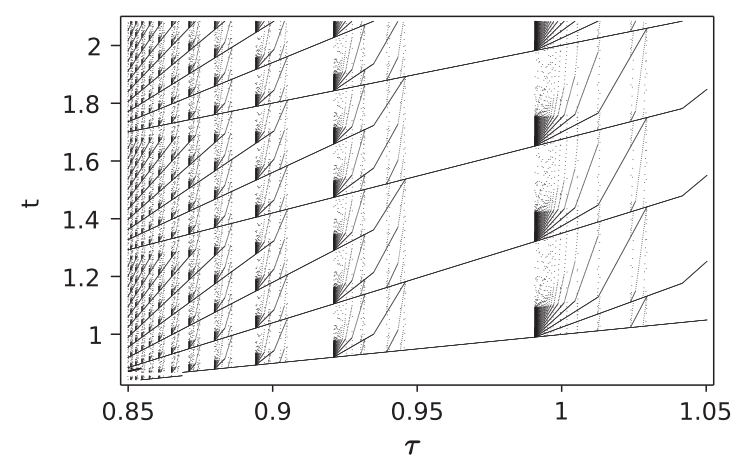

FIG. 5. Bifurcation diagrams of graph nodes for varying $\tau$ and fixed $f=3 / 5$ and $\sigma=1 / 2$. Each point corresponds also to a graph node parametrized by the variable $t\left(x_{1}, \kappa_{1}\right)$.

$\mathcal{F}_{0,1}$ (see Appendix D). Therefore, the behavior of the physical observables reflects the underlying complexity of $\mathcal{F}_{0,1}$ orbits.

In addition to the inherent complexity of piecewise linear circle maps, since the action of the two maps here involved does not commute, the combined evolution may generate new dynamical scenarios. In particular, we remark that neither of the two maps $\mathcal{F}_{0,1}$ is chaotic separately, due to the segments with zero slope that reset trajectories. However, the alternation of the two maps can give rise to trajectories that avoid falling into the zero-slope segments and fall repeatedly on linear pieces with slope larger than 1 , which are typically associated to chaos [22]. Indeed, when $f \tau<1-\sigma, 2 \sigma /(1-f)<\tau<$ $2 \sigma /[f(1-f)]$ and $1-\sigma<\tau$ are satisfied (e.g., for $f<1 / 3$ in Fig. 3), there exist arbitrarily long strings $\alpha_{i}$ that correspond to repeated mapping with a constant slope $\frac{1+(1-f)^{2}}{2(1-f)^{2}}>1$. The Lyapunov exponent of the corresponding chaotic trajectory can be shown to be strictly larger than 1 (see Appendix E). Accordingly, in this parameter regime, the evolution of the system may be seen as a sequence of fragments with a chaotic evolution that are reset to the initial condition after a finite random time, what we call piecewise chaotic dynamics.

While chaos may not be apparent for short trajectories, the signature of this hidden chaos will manifest in the sensitivity to physical parameters of long trajectories [22] and on the statistical properties of node distributions. Figure 6 shows the

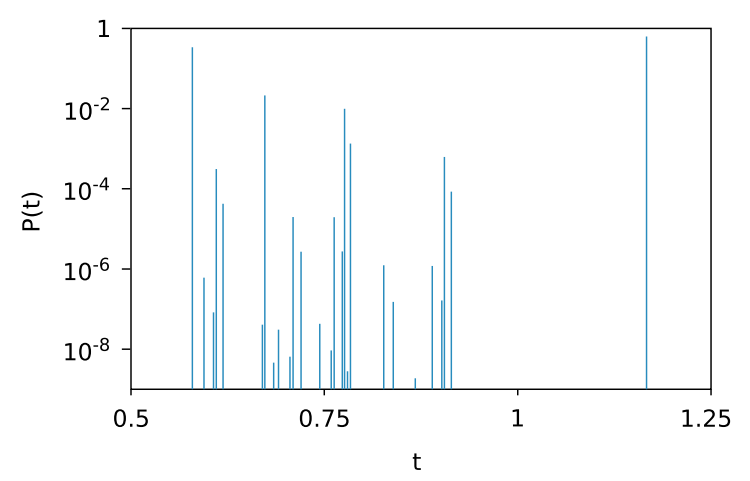

FIG. 6. Probability distribution to visit a graph node for $(\tau, \sigma, f)=(3 / 2,1 / 2,1 / 7)$. Node position is parametrized with the variable $t\left(x_{1}, \kappa_{1}\right)$.

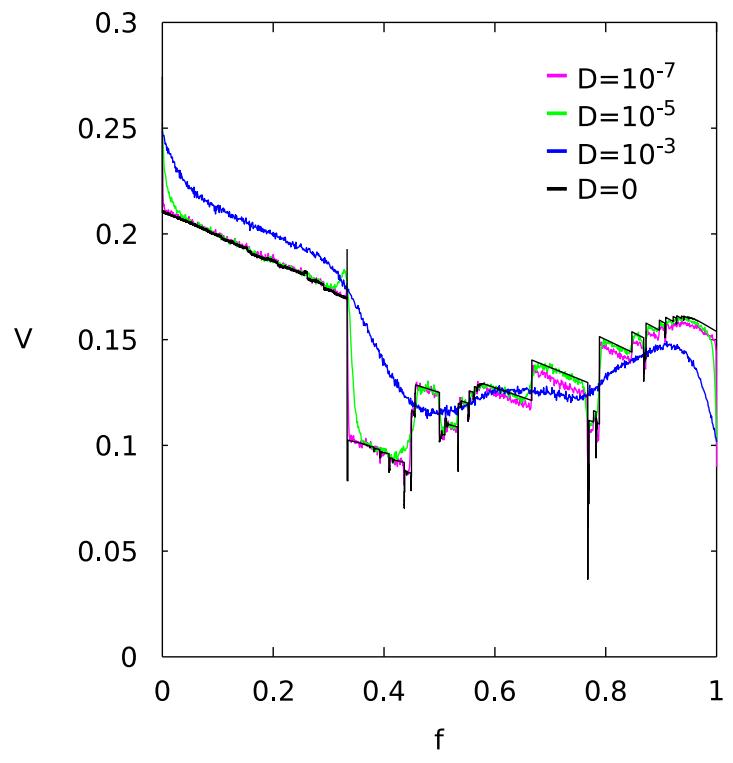

FIG. 7. Velocity-force $V(f)$ curve for $\tau=3 / 2$ and $\sigma=1 / 2$. The black curve corresponds to the exact $V(f)$ at the vanishing-noise limit Fig. 3. The red, blue, and green curves correspond to the mean $V(f)$ for two interacting motors with finite noise intensities $D=10^{-7}, D=10^{-5}$, and $D=10^{-3}$, respectively.

probability distribution to visit a graph node, parametrized by the function $t\left(x_{1}, \kappa_{1}\right)$. We find that the values of $t\left(x_{1}, \kappa_{1}\right)$ are very heterogeneously disperse, with gaps that are never visited and regions with high density, similarly to what would be characteristic of chaotic systems. Furthermore, in Appendix E, we show another signature of chaos that is associated to how such distributions are increasingly sensitive to parameter variations for larger trajectories.

\section{DISCUSSION}

In this study, we have unveiled unsuspected complexity for the vanishing-noise coupled-ratchet problem. In addition to the fundamental interest of having a nontrivial exactly solvable model, and of the new dynamical scenarios identified, it is worth enquiring to what extent the unveiled features subsist when there is a small but finite noise. To this end, we have simulated Eq. (1) with decreasing but finite noise intensity Fig. 7. We show that the irregular features of the limit solution are progressively resolved as the noise intensity decreases, exhibiting for instance smooth but nonmonotonic behavior of $V(f)$ at finite noise, a phenomenon difficult to understand without the knowledge gained from the limiting case. This illustrates that signatures of the complexity here unveiled should be experimentally observable in more realistic scenarios.

As a final comment, we stress the appealing ways in which this problem combines concepts such as ratchets, random walks, complex networks, circle maps, chaos, or fractals, and in particular the new scenario of piecewise chaos, which may hopefully be inspiring for further theoretical and experimental research. 


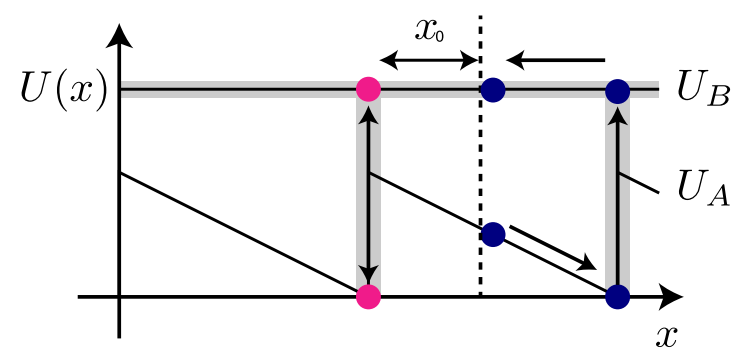

FIG. 8. Definition of the accessible configurations for particle 1. The trailing load-free motor is shown in pink and the leading motor that is subjected to an external load $f$ is shown in blue. $x_{0}$ represents the maximal distance that the leading motor can travel in state B without being in contact with the trailing motor.

\section{ACKNOWLEDGMENTS}

Financial support from Spanish Ministry of Economy and Competitiveness is acknowledged (Project No. FIS201678507-C2-2-P) and from the Generalitat de Catalunya (Project No. 2017-SGR-1061). C.B.-M. acknowledge financial support from the University of Geneva.

\section{APPENDIX A}

Here we introduce a convenient one-dimensional parametrization of graph nodes. All configurations of the leading motor (or particle 1) can be mapped into a single degree of freedom $t\left(x_{1}, \kappa_{1}\right)$ that is defined as the time $t$ required for particle 1 to reach $\left(x_{1}, \kappa_{1}\right)$ starting at $\left(x_{0}, 1\right)$ without being in contact with particle 2. For simplicity the present analysis is restricted to the parameter domain $f \tau<1-\sigma$ and $1-\sigma<\tau$ or the parameter domain $f \tau>1-\sigma$; otherwise, some of the definitions here presented need to be adapted.

Figure 8 shows a schema of a typical cyclic trajectory of the leading particle. The interval $\left[x_{0}, 1\right]$ defines what we call accessible configurations for particle 1 , that is, those configurations for which the trailing motor (or particle 2) can undergo a transition (e2). The maximum of $t$ is $T=t\left(x_{0}, 0\right)=$ $\left(1-x_{0}\right) /[f(1-f)]$. When $f \tau<1-\sigma$, we set $x_{0}=1-f \tau$ and $T=\tau /(1-f)$, whereas in the opposite regime $f \tau>$ $1-\sigma, x_{0}=\sigma$ and $T=(1-\sigma) /[f(1-f)]$. In the former case, particle 1 undergoes periodic cycles of period $T$. In the latter, particle 1 could enter the region $x_{1}<x_{0}$ but displacing particle 2, thus impeding an (e2) transition. With the above definitions, the accessible configurations for particle 1 are constrained within the interval $\left(x_{1}, \kappa_{1}\right) \in\left[x_{0}, 1\right] \times\{0,1\}$.

The mapping $t\left(x_{1}, \kappa_{1}\right)$ can be computed explicitly and reads

$$
t\left(x_{1}, \kappa_{1}\right)=\left\{\begin{array}{cl}
\frac{x_{1}-x_{0}}{1-f} & \kappa_{1}=1 \\
\frac{1-x_{0}}{1-f}+\frac{1-x_{1}}{f} & \kappa_{1}=0
\end{array} .\right.
$$

\section{APPENDIX B}

Here we show that the velocity-force curve is continuous in a dense subset of parameters. First, we restrict the parameter space to $f \tau<1-\sigma, \tau<2 \sigma /(1-f)$, and $1-\sigma<\tau$. If the graph topology is constant, then path summations of graphs will reflect the continuity of path weights to model parameters, such as the duration or the distance between two nodes. Hence, the continuity of the velocity-force curve reduces to finding a parameter set with the same graph topology.

When $f$ is irrational, the system dynamics can be captured by a random walker in an infinite graph. Using the parametrization for node graphs introduced in Appendix A, $t\left(x_{1}, \kappa_{1}\right)$ with period $T=\tau /(1-f)$, the node set $\mathcal{N}$ reads

$$
\mathcal{N}=\left\{t=\tau-\frac{1-f \tau-\sigma}{1-f}+n \tau(\bmod T) \mid n \in \mathbb{N}\right\} .
$$

The configuration for which particle 1 undergoes an event (e2) need to be discarded from $\mathcal{N}$. Graphs that include this configuration are determined by the condition

$$
m_{1} \tau=m_{2} T+\frac{(1-\sigma) f}{1-f}+1-f
$$

where $m_{1}$ and $m_{2}$ are a pair of integers. For example, Eq. (B2) is fulfilled by the triad $(\tau, \sigma, f)=(1,1 / 3,1 / 3)$. Let us define the parameter subset $\mathbb{M}$ that satisfy Eq. (B2). The graphs at forces $f \in \mathbb{R} /(\mathbb{Q} \cup \mathbb{M})$ preserve topology, that is, for irrational force values that unfulfill Eq. (B2). Because $\mathbb{M}$ and $\mathbb{Q}$ are numerable sets, then $\mathbb{R} /(\mathbb{Q} \cup \mathbb{M})$ is dense. Therefore, the velocity-force curve is continuous in a dense subset of parameters.

\section{APPENDIX C}

Here we compute analytically the circle maps $\mathcal{F}_{0}$ and $\mathcal{F}_{1}$ for some specific parameter regimes. An exhaustive derivation of all circle maps present in our problem falls beyond the scope of this study.

A circle map is a transformation from a circle into itself. In our case, the interval $\left(x_{1}, \kappa_{1}\right) \in\left[x_{0}, 1\right] \times\{0,1\}$ is homeomorphic to a circle because of the one-dimensional parametrization $t=t\left(x_{1}, \kappa_{1}\right)$ (see Appendix A for definitions). As explained in the main text, the circle maps $\mathcal{F}_{0}$ and $\mathcal{F}_{1}$ are defined by the deterministic evolution between two successive stochastic events (e2) depending on whether particles 2 switches to $A\left(\kappa_{2}=1\right)$ or remains at $B\left(\kappa_{2}=0\right)$, respectively.

$\mathcal{F}_{0}$ corresponds to the circle map for which the outcome of the event (e2) for particle 2 is $\kappa_{2}=0$. In the parameter regime $f \tau<1-\sigma$ (e.g., for $f<1 / 3$ in Fig. 3 ), the leading particle in state $B$ can not interact with the trailing particle at the boundary discontinuity, and the corresponding circle map takes the form

$$
\mathcal{F}_{0}(t)=t+\tau \quad 0<t \leqslant T,
$$

where $T=\tau /(1-f) . \mathcal{F}_{0}$ is formally equivalent to the circle map of a system composed by two uncoupled oscillators with natural periods $T$ and $\tau$, Fig. 4(a). The circle map given by Eq. (C1) features periodic or quasiperiodic orbits, depending on whether the dimensionless force $f$ is a rational number or an irrational number, respectively. Trajectories are $n$-periodic orbits, when $n \tau=m T$, being $n$ and $m$ integer numbers. The period of such orbits equals the denominator of the rational $f$.

In the regime $f \tau>1-\sigma$ and $T>2 \tau$ [e.g., for $f>(3+$ $\sqrt{3}) / 6$ in Fig. 3], the leading particle in state $B$ may push the trailing particle away from the boundary discontinuity. In this 
situation, $\mathcal{F}_{0}$ with period $T=(1-\sigma) /[f(1-f)]$ reads

$$
\mathcal{F}_{0}(t)=\left\{\begin{array}{cc}
t+\tau & 0<t \leqslant T-\tau \\
\tau+g_{0}(t) & T-\tau<t \leqslant g_{1} \\
\tau & g_{1}<t \leqslant g_{2} \\
\tau+\left(t-g_{2}\right) & g_{2}<t \leqslant T
\end{array}\right.
$$

where $\quad g_{0}(t)=\max \left\{\left[1+(1-f)^{2}\right]\left(t-g_{1}\right) /\left[2(1-f)^{2}\right]+\right.$ $\tau, 0\}, g_{1}=(2-f) f T-f \tau$, and $g_{2}=\min (T,\{f[T(2-f)$ $(1-f)+f \tau]\} /[2(1-f)])$. The circle map given by Eq. (C2) is piecewise linear with an intermediate flat segment, Fig. 4(c). These type of maps feature a special set of periodic orbits with a point at the flat segment, which is referred in the literature of circle maps as superstable orbits, since their Lyapunov exponent is exactly zero [20,21]. In spite of the apparent simplicity of Eq. (C2), its bifurcation diagram can exhibit mode-locking or complex self-similar structures Fig. 9(a), such as the so called nested period incrementing bifurcation scenario $[20,21]$.

$\mathcal{F}_{1}$ corresponds to the circle map for which the outcome of the event (e2) for particle 2 is $\kappa_{2}=1$. In the regime, where both $f \tau<1-\sigma, \tau<2 \sigma /(1-f)$ and $1-\sigma<\tau$ are satisfied (e.g., no $f$ in Fig. 3), the corresponding circle map with period $T=\tau /(1-f)$ reads

$$
\mathcal{F}_{1}\left(t^{\prime}\right)=T-\frac{1-\sigma}{1-f} \quad 0<t^{\prime}<T,
$$

where $t^{\prime}=\bmod [t+1-(\sigma+f \tau), T] . \mathcal{F}_{1}$ consists of a single flat segment with a unique stable fixed point Fig. 4(b). This fixed point corresponds to the graph node origin, that is, the first node encountered after the configuration $\left(x_{1}, x_{2}, \kappa_{1}, \kappa_{2}\right)=\left(1^{-}, 1^{-}-\sigma, 1,1\right)$.
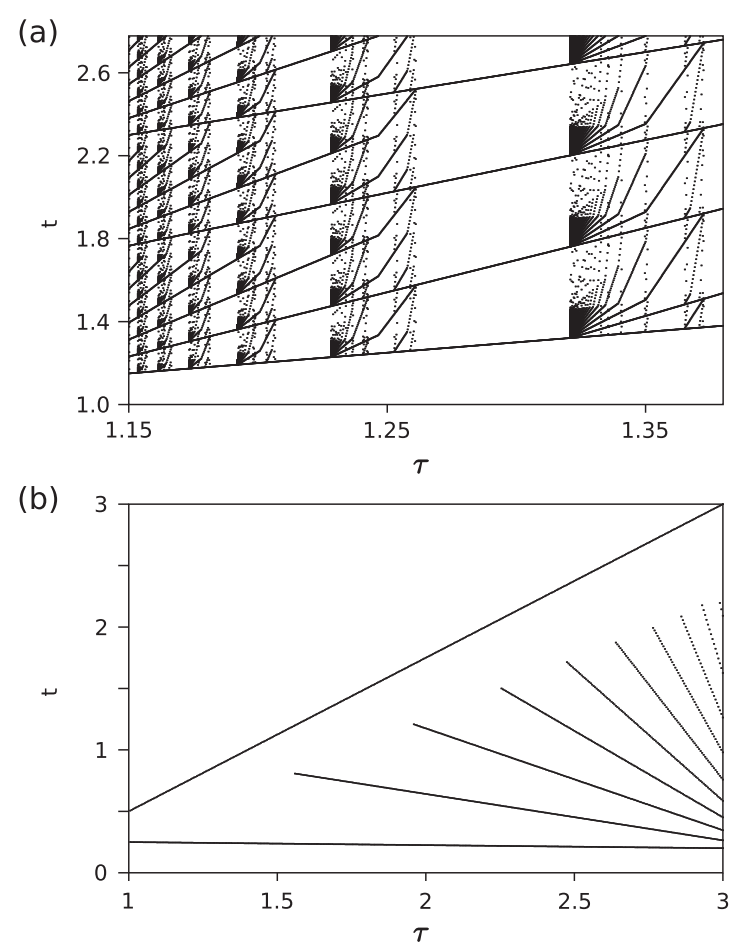

FIG. 9. (a) Bifurcation diagram of $\mathcal{F}_{0}$ given by Eq. (C2) for varying $\tau$ with $\sigma=1 / 3$ and $f=2 / 3$. (b) Bifurcation diagram of $\mathcal{F}_{1}$ given by Eq. (C5) for varying $\tau$ with $\sigma=2 / 5$ and $f=1 / 5$.
In the regime, where both $f \tau>1-\sigma, \tau<2 \sigma /(1-f)$ and $\tau<T$ are satisfied (e.g., $f>1 / 3$ in Fig. 3), the corresponding circle map with period $T=(1-\sigma) /[f(1-f)]$ reads

$$
\mathcal{F}_{1}(t)=\tau \quad 0<t<T .
$$

$\mathcal{F}_{1}$ is a single flat segment with a unique stable fixed point that corresponds to the graph origin, akin to Eq. (C3).

In the regime where both $f \tau<1-\sigma, 2 \sigma /(1-f)<\tau<$ $2 \sigma /[f(1-f)]$ and $1-\sigma<\tau$ are satisfied (e.g., for $f<1 / 3$ in Fig. 3), $\mathcal{F}_{1}$ takes the form

$$
\mathcal{F}_{1}\left(t^{\prime}\right)=\left\{\begin{array}{cc}
\sigma \frac{2-2 f+f^{2}}{(1-f)^{2}}+\tau \frac{2-2 f-f^{2}}{2(1-f)}-g_{5} & 0<t^{\prime} \leqslant f^{2} T \\
2 \tau+\frac{1+(1-f)^{2}}{2(1-f)^{2}}\left(t-g_{3}\right)-g_{5} & f^{2} T<t^{\prime} \leqslant g_{3} \\
\tau-g_{5} & g_{3}<t^{\prime} \leqslant g_{4} \\
\sigma \frac{2-2 f+f^{2}}{(1-f)^{2}}+\tau \frac{2-2 f-f^{2}}{2(1-f)}-g_{5} & g_{4}<t^{\prime} \leqslant T
\end{array}\right.
$$

where $t^{\prime}=\bmod [t+1-(\sigma+f \tau), T], \quad T=\tau /(1-f)$, $g_{3}=T\left[(1-f)^{2}+f^{2}\right]-2 \sigma, \quad g_{4}=g_{3}+2 \sigma, \quad$ and $g_{5}=$ $(1-\sigma-f \tau) /(1-f)$. The circle map given by Eq. (C5) is piecewise linear with two distinct flat segments Fig. 4(d). Its superstable orbits have at least one point in either of the two flat segments, and its bifurcation diagram can exhibit mode locking and signatures of the period incrementing scenario [20,21], as shown in Fig. 9(b).

\section{APPENDIX D}

Here we provide an illustrative example of the connection between circle-map orbits and physical observables from our two-motor problem. To this end, we compute periodic orbits
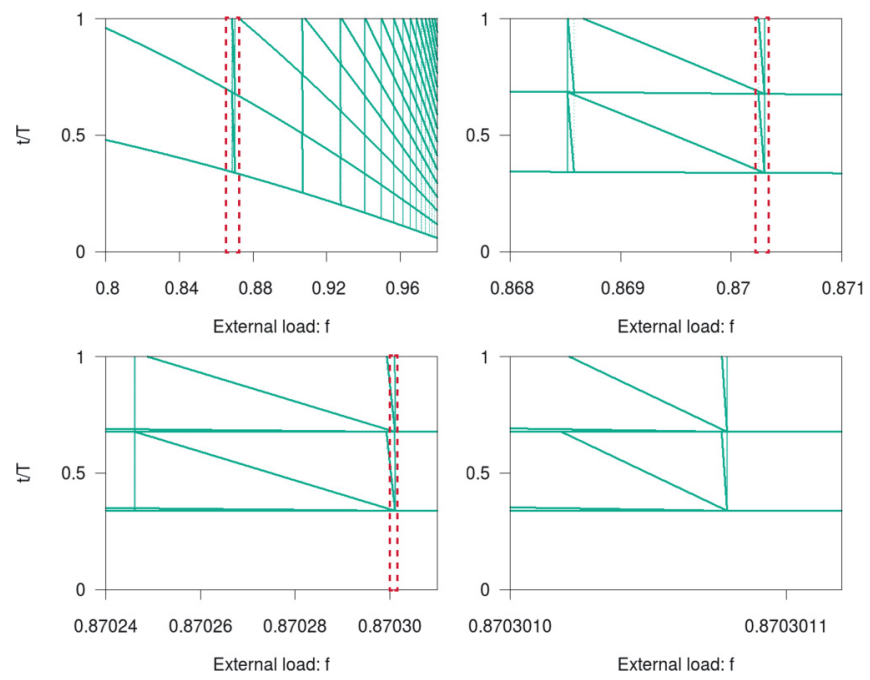

FIG. 10. Bifurcation diagram of the circle map $\mathcal{F}_{0}$ given by Eq. (C2) for $f \in(4 / 5,1), \sigma=1 / 2$ and $\tau=3 / 2$ (e.g., for $f>4 / 5$ in Fig. 3). Each panel shows graph nodes parametrized by the variable $t\left(x_{1}, \kappa_{1}\right)$ that is normalized by $T=(1-\sigma) /[f(1-f)]$ varying the external load $f$. From left to right and top to bottom, the range of forces decreases, and each panel zooms in the self-similar structure laying at force values that are marked approximately in a red rectangle. 
that emerge from the alternation of the circle maps $\mathcal{F}_{0}$ and $\mathcal{F}_{1}$ and compare their structure with the corresponding domain from the velocity force curve $V(f)$ displayed in Fig. 3. In particular, we focus our analysis within the regime $f \in(4 / 5,1)$, $\sigma=1 / 2$, and $\tau=3 / 2$. In this regime, the circle map given by Eq. (C4) is $\mathcal{F}_{1}=$ const; consequently, the periodic orbits arising from the alternation of both $\mathcal{F}_{0}$ and $\mathcal{F}_{1}$ are exactly the same as the periodic orbits of $\mathcal{F}_{0}$ given by Eq. (C2) that in addition contain $\mathcal{F}_{1}=$ const. Figure 10 shows the corresponding bifurcation diagram at increasing levels of force resolution. The leftmost panel displays characteristic signatures of the so-called nested period incrementing scenario in the context of circle maps, with a progressive increase of the number of nodes at increasing forces and complex arrangements of nodes at narrow force windows. When we zoom in at one of these force windows, we observe that nodes seem to arrange in a self-similar way, a characteristic feature of such scenario. Self-similar structures (i.e., staircase-like shapes) are also found in the velocity force curve from Fig. 3 within the same parameter range $f \in(4 / 5,1), \sigma=1 / 2$, and $\tau=3 / 2$. Therefore, this analysis illustrates the deep connection between the periodic orbits of $\mathcal{F}_{0,1}$, and the behavior of physical observables in the two-motor problem.

\section{APPENDIX E}

Here we discuss a set of sufficient conditions, for which the two-motor problem exhibits chaos.

As discussed in the main text, a dynamical trajectory can be mapped into a string of zeros and ones corresponding to the iteration of the circle maps $\mathcal{F}_{0}$ and $\mathcal{F}_{1}$. In the regime where both $f \tau<1-\sigma, 2 \sigma /(1-f)<\tau<2 \sigma /[f(1-f)]$ and $1-$ $\sigma<\tau$ are satisfied (e.g., for $f<1 / 3$ in Fig. 3), $\mathcal{F}_{0}$ is given by Eq. (C1) and $\mathcal{F}_{1}$ is given by Eq. (C5). Each circle map separately does not generate chaos, however, the combination of both circle maps may give rise to chaos.

$\mathcal{F}_{0}$ feature an infinite number of $n$-periodic orbits, if and only if $f$ is a rational number (see Appendix $\mathrm{C}$ for further details). Furthermore, the period of such orbits is given by the denominator of $f$ [i.e., $n=\operatorname{den}(f)]$. Then, we construct the following multivaluated circle map

$$
\mathcal{F}(t)=\left\{\mathcal{F}_{1} \circ \mathcal{F}_{0}^{(1)}(t), \ldots, \mathcal{F}_{1} \circ \mathcal{F}_{0}^{(\operatorname{den}(f))}(t)\right\} .
$$

Each component of $\mathcal{F}$ either lies into one of the two flat segments in Eq. (C5) or into the positive-slope segment in Eq. (C5). If $g_{3}-f^{2} T>T / \operatorname{den}(f)$, then it exists an integer $i$ such that $\mathcal{F}_{0}^{(i)}(t) \in\left(f^{2} T, g_{3}\right)$ for every $0<t<T$. In other words, the domains of positive slope cover the whole interval of $t$, because the positive-slope segments partially overlap, as shown for example in Fig. 11. Then, there is at least one of the components of the multivalued map Eq. (E1), that has finite-slope for any $t$. Since each component corresponds to a certain iteration of the original maps, it is then guaranteed that there is a specific trajectory that corresponds to an effective mapping with just positive-slope segments. A piecewise linear discontinuous map with a constant slope larger than one generates chaos, and its slope determines the corresponding Lyapunov exponent $\lambda$.

The Lyapunov exponent $\lambda$ from Eq. (E1) is bounded from above and below. The upper bound is obtained by assuming

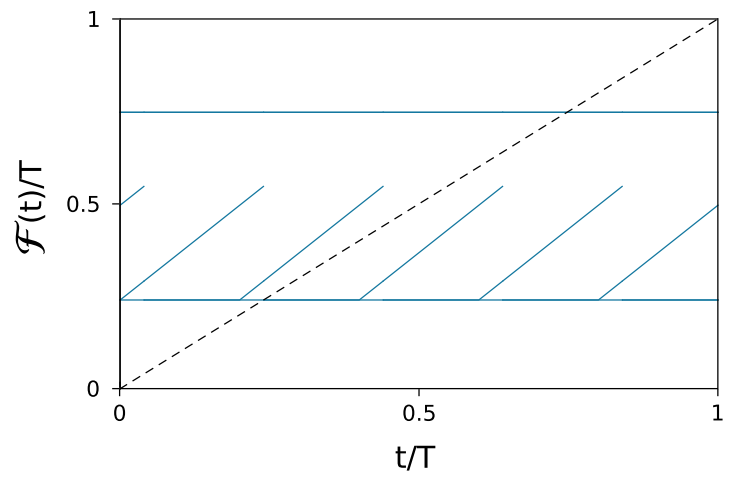

FIG. 11. Multivalued circle map given by Eq. (E1) for $(\tau, \sigma, f)=(2,1 / 2,1 / 5)$. Both variables $\mathcal{F}$ and $t$ are normalized by the corresponding period $T=\tau /(1-f)$.

that on every iteration of the circle map $\mathcal{F}_{1}$ in the chaotic domain no iterations of the circle map $\mathcal{F}_{0}$ are required, hence

$$
\lambda(f)<\frac{1}{2}+\frac{1}{2(1-f)^{2}},
$$

The lower bound is obtained by assuming that on every iteration of the circle map $\mathcal{F}_{1}$ in the chaotic domain, it is required to iterate the circle map $\mathcal{F}_{0}$ the maximal number of times $\operatorname{den}(f)-1$. In conclusion, the Lyapunov exponent

$$
1<1+\frac{1}{\operatorname{den}(f)}\left[-\frac{1}{2}+\frac{1}{2(1-f)^{2}}\right]<\lambda(f),
$$

is greater than 1 , rendering chaotic long trajectories. This result implies that the time evolution of the system is composed of fragments with chaotic dynamics. Equations (E2) and (E3) depend on the dimensionless force $f$ only. Long trajectories carry in general a small probability, thus the signatures of chaos will not be visible by comparing trajectories with similar initial conditions, but by the sensitivity to parameters of the long chaotic sequences.

Another way to view the underlying chaotic dynamics is by constructing the probability of visiting the different nodes in the graph. Figure 12 shows that, even though the statistics is dominated by a relatively reduced number of nodes, the peak

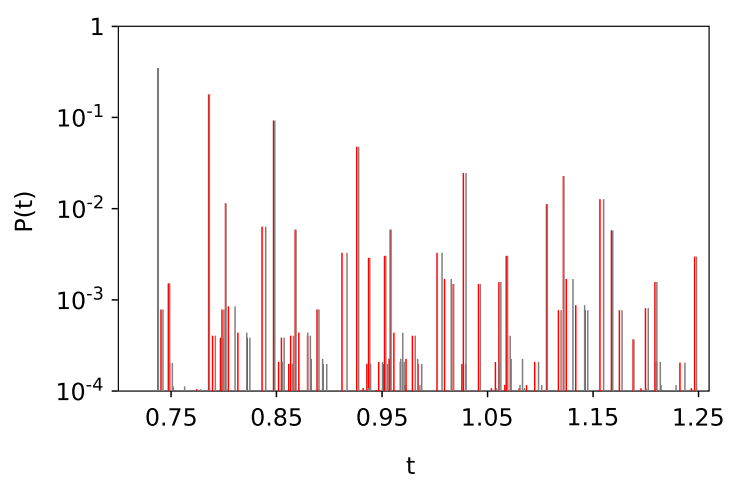

FIG. 12. Histogram of the probability distribution to visit graph nodes for $\tau=1, f=1 / 5$, and $\sigma=1 / 5$ (black) and $\sigma=1 / 5+$ $1 / 10000$ (red). 
positions, which define the nodes visited, are distributed very heterogeneously, with some gaps and some regions of higher density, as corresponding to a chaotic system.

Figure 12 also shows that two adjacent peaks from two histograms with an infinitesimal change in parameter sets, are further away from each other, the smaller their peaks are. This is a signature of the sensitivity of the dynamics to parameters, suggesting that for the same sequence of iterations of $\mathcal{F}_{0,1}$, the resulting trajectories will diverge from each other exponentially.
[1] F. Jülicher, A. Ajdari, and J. Prost, Rev. Mod. Phys. 69, 1269 (1997).

[2] J. M. R. Parrondo, G. P. Harmer, and D. Abbott, Phys. Rev. Lett. 85, 5226 (2000).

[3] J. G. Orlandi, C. Blanch-Mercader, J. Brugues, and J. Casademunt, Phys. Rev. E 82, 061903 (2010).

[4] C. Leduc, O. Campàs, K. B. Zeldovich, A. Roux, P. Jolimaitre, L. Bourel-Bonnet, B. Goud, J.-F. Joanny, P. Bassereau, and J. Prost, Proc. Natl. Acad. Sci. USA 101, 17096 (2004).

[5] D. Oriola, S. Roth, M. Dogterom, and J. Casademunt, Nat. Commun. 6, 8025 (2015).

[6] O. Campàs, Y. Kafri, K. B. Zeldovich, J. Casademunt, and J.-F. Joanny, Phys. Rev. Lett. 97, 038101 (2006).

[7] G. Koster, M. VanDuijn, B. Hofs, and M. Dogterom, Proc. Natl. Acad. Sci. USA 100, 15583 (2003).

[8] D. Oriola and J. Casademunt, Phys. Rev. Lett. 111, 048103 (2013).

[9] O. Campàs, C. Leduc, P. Bassereau, J. Casademunt, J.-F. Joanny, and J. Prost, Biophys. J. 94, 5009 (2008).

[10] P. Hänggi and F. Marchesoni, Rev. Mod. Phys. 81, 387 (2009).

[11] B. J. Lopez, N. J. Kuwada, E. M. Craig, B. R. Long, and H. Linke, Phys. Rev. Lett. 101, 220601 (2008).
[12] S. Matthias and F. Müller, Nature (London) 424, 53 (2003).

[13] L. Gorre-Talini, J. P. Spatz, and P. Silberzan, Chaos 8, 650 (1998).

[14] S.-H. Lee and D. G. Grier, J. Phys.: Condens. Matter 17, S3685 (2005).

[15] L. P. Faucheux, L. S. Bourdieu, P. D. Kaplan, and A. J. Libchaber, Phys. Rev. Lett. 74, 1504 (1995).

[16] P. Tierno, S. V. Reddy, T. H. Johansen, and T. M. Fischer, Phys. Rev. E 75, 041404 (2007).

[17] A. Sokolov, M. M. Apodaca, B. A. Grzybowski, and I. S. Aranson, Proc. Natl. Acad. Sci. USA 107, 969 (2010).

[18] See Supplemental Material at http://link.aps.org/supplemental/ 10.1103/PhysRevE.101.012203 for additional information about supplemental Movies 1-3.

[19] V. Avrutin and M. Schanz, Nonlinearity 19, 531 (2006).

[20] B. Schenke, V. Avrutin, and M. Schanz, in Proceedings of the Royal Society of London A: Mathematical, Physical and Engineering Sciences (The Royal Society, 2011), Vol. 467, pp. 1503-1518.

[21] B. Futter, V. Avrutin, and M. Schanz, Chaos Solitons Fractals 45, 465 (2012).

[22] H. G. Schuster and W. Just, Deterministic Chaos: An Introduction (John Wiley \& Sons, New York, 2006). 